\title{
Artery of Percheron infarct: hiding in plain sight
}

\author{
Authors: Charles Badu-Boateng, ${ }^{\mathrm{A}}$ Dhaarica Jeyanesan, ${ }^{\mathrm{A}}$ Jonathan Easaw ${ }^{\mathrm{A}}$ and Mark Lloyd ${ }^{\mathrm{B}}$
}

\begin{abstract}
A 54-year-old woman presented to the emergency department with fluctuating consciousness without localising signs and non-specific neurology. Urgent computed tomography (CT) was unremarkable and differentials of opioid overdose or post-ictal state were considered. Magnetic resonance imaging of the brain demonstrated an artery of Percheron (AOP) infarct; resulting from occlusion of an uncommon anatomical variant in the thalamic and midbrain circulation. This should be considered in patients presenting with fluctuating consciousness and normal CT.
\end{abstract}

KEYWORDS: stroke, artery of Percheron, fluctuating consciousness, infarct

DOI: 10.7861/clinmed.2021-0383

\section{Introduction}

Artery of Percheron (AOP) infarcts are estimated to account for about $0.1 \%-2 \%$ of ischaemic strokes, rising to $4 \%-35 \%$ of thalamic strokes. ${ }^{1}$ Detecting an acute presentation of AOP infarct can prove difficult without magnetic resonance imaging (MRI). Although an uncommon presentation of a stroke, clinicians should consider it as a differential to permit timely diagnosis and subsequent therapy.

\section{Case presentation}

A 54-year-old woman was brought in by ambulance with history of slurred speech and collapse. Initial paramedic assessment found her to be unresponsive with a Glasgow Coma Score (GCS) of 3. Her GCS improved on transit to the hospital and was 15 by arrival. Neurological examination was normal. Initial vital signs showed respiratory rate 17 breaths per minute, pulse at 83 beats per minute, temperature of $36.1^{\circ} \mathrm{C}$, blood pressure of $152 / 91$ $\mathrm{mmHg}$, oxygen saturation of $100 \%$ and electrocardiography showed sinus rhythm.

During stroke team assessment, the patient experienced sudden onset slurred speech, right sided weakness and diplopia, with reduction in her GCS to 8: E1, V2 and M5. Her pupils were unequal

Authors: ${ }^{\text {A }}$ senior house officer, Frimley Park Hospital, Camberley,

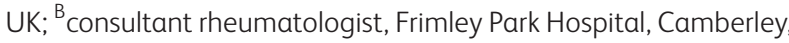
UK (right $2 \mathrm{~mm}$ and left $3 \mathrm{~mm}$ ); and she tolerated a nasopharyngeal airway adjunct.

Concurrent collateral history suggested possible over-medication with 'two strips of the $30 \mathrm{mg} / 500 \mathrm{mg}$ co-codamol tablets' reportedly taken overnight. Cardiovascular risk factors included smoking and excess alcohol consumption ( 140 units/week). Other past medical history of note included clinically stable bipolar disorder and history of and previous intentional medication overdose. Medications were venlafaxine, mirtazapine and co-codamol. A trial of naloxone was ineffective. Urgent non-contrast computed tomography (CT) of the head was reported as unremarkable for acute infarct or haemorrhage (Fig 1a), and CT angiography of her aorta and bilateral carotid arteries was reported as unremarkable for significant stenosis (Fig 1b and c).

Admission full blood count, renal function test, liver function test, C-reactive protein and arterial blood gas were normal. Toxicology screen was negative.

Due to her fluctuating GCS and non-specific neurological signs, she was referred to the medical team for further investigation as stroke was deemed unlikely. She was started on intravenous Pabrinex, and loaded on levetiracetam for possible non-convulsive seizure and transferred to a medical high dependency unit for supportive care.

Her GCS later increased to 9 (E1, V3 and M5) with positional snoring and no airway compromise. Her left pupil was fixed and dilated and she had normal power and tone in all four limbs. By the afternoon, GCS was 11 (E2, V4 and M5) and was able to cough to protect her airway.

Neurology review noted a horizontal dysconjugate gaze, with brisk reflexes in her right upper and lower limbs with an associated up-going plantar on her right side. Differential diagnoses included possible underlying vasoactive substance effects, deep cerebral venous thrombosis and top of basilar artery syndrome. MRI of the brain was recommended.

Over a 24-hour period, her GCS improved to 15, although she was unable to recall why she was in hospital. She denied taking any medication overdose prior to admission. Her left pupil remained fixed and dilated, with evidence of internuclear ophthalmoplegia (INO) on looking to her right, and she also had left sided partial ptosis.

MRI of the brain subsequently showed brainstem and bilateral thalamic stroke secondary to AOP infarct (Fig 2). Echocardiography showed non-dilated left ventricle with preserved ejection fraction ( $55 \%-60 \%)$ and impaired relaxation. Right ventricular function was normal and she had normal bi-atrial size. 
Fig 1. a) Computed tomography of the head, unremarkable for acute infarct or haemorrhage. $b$ and c) Computed tomography angiography of the aorta and bilateral carotid arteries, unremarkable for significant stenosis.
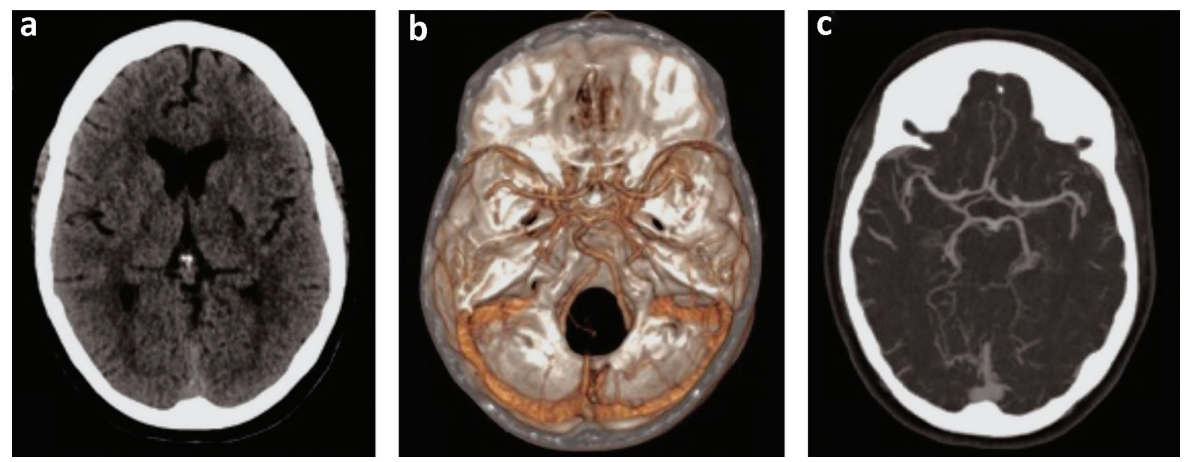

\section{Outcome and follow-up}

On confirmation of ischaemic stroke, $300 \mathrm{mg}$ aspirin daily was started for 2 weeks, followed by long-term $75 \mathrm{mg}$ clopidogrel. She was a started on a proton pump inhibitor and a statin as per National Institute for Health and Care Excellence (NICE) guidelines; and discharged to community rehabilitation 6 days after admission.

Residual neurology on discharge included left INO and diplopia. Power and tone in all four limbs were normal.

\section{Discussion}

The AOP was first described in 1973. It is a single arterial trunk originating from the proximal posterior cerebral artery. It supplies the bilateral paramedian thalamus and rostral midbrain. AOP infarcts cause bilateral paramedian thalamic infarcts with or without midbrain involvement, and the profound behavioural changes can make it a diagnostic challenge. ${ }^{2}$

Despite presenting as a stroke call, the patient's fluctuating GCS, lack of complete history coupled with unremarkable CT of the brain and $\mathrm{CT}$ angiography led attention away from stroke as a possible cause of her symptoms. This diagnostic delay also precluded our patient from being considered for appropriate thrombolytic therapy as per NICE guidelines despite arriving into hospital within 4 hours of symptom onset, with no evidence of cerebral haemorrhage on emergency un-enhanced CT of the brain. ${ }^{3}$
Previous studies and reports have also suggested that diffusionweighted MRI is the best modality for detecting acute AOP infarcts, although interval scans maybe required in cases of high clinical suspicion despite negative initial MRI brain. A negative cerebral angiography can give false reassurance as the AOP is an uncommon anatomical variant, and therefore its absence may not necessarily raise concern. A high index of suspicion is therefore warranted when AOP infarct is suspected. ${ }^{4}$

Our patient was noted to have unilateral INO following infarct of her AOP. INO results from damage to the interneuron between two nuclei of cranial nerves VI and III (internuclear) known as the medial longitudinal fasciculus (MLF). INO typically manifests in young multiple sclerosis patients, or in adults as brainstem infarcts. Less common causes include trauma, tentorial herniation, infection, tumour, iatrogenic injury, haemorrhage and vasculitis and can often be an isolated or the predominant symptom of brainstem lesions. Rostral brainstem lesions also typically affect levels of alertness, in addition to behavioural changes, pupillary and oculomotor defects as well as bilateral INO. ${ }^{3}$

As with all acute ischaemic strokes, early thrombolysis in patients with no contra-indications offers the best prognosis in an AOP infarct. $^{2}$

\section{Key points}

> AOP infarct should be considered in a patient presenting with altered consciousness and changing neurology, once other
Fig 2. Magnetic resonance imaging of the brain showing brainstem and bilateral thalamic stroke secondary to artery of Percheron infarct.
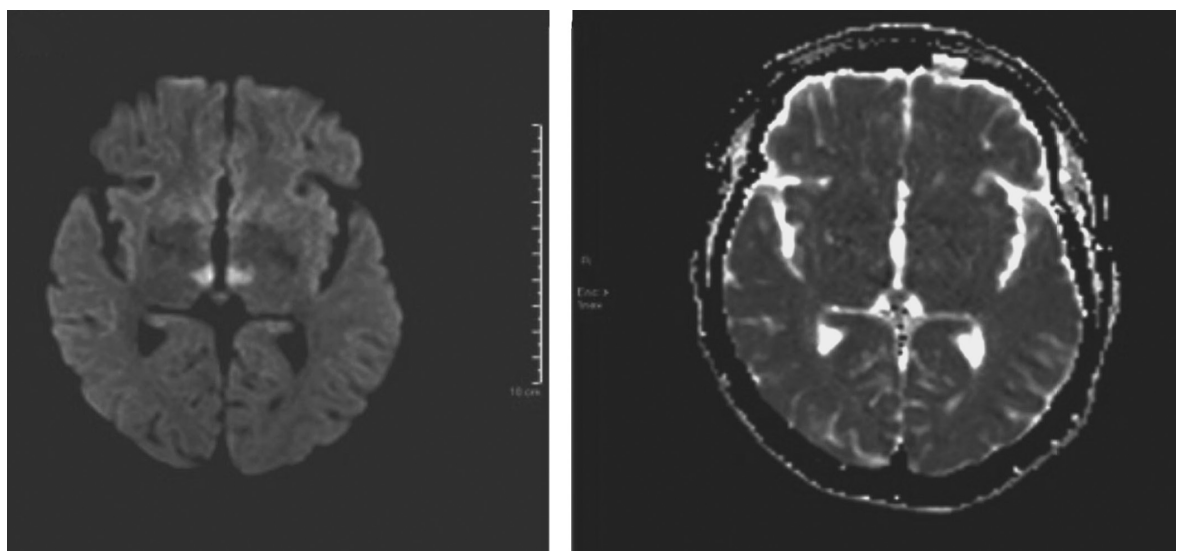
causes, including seizures, drug toxicity, infection and multiple sclerosis have been excluded.

> Diffusion-weighted MRI is the best modality for diagnosing acute AOP infarcts and a high index of suspicion is required when initial MRI or conventional angiography is unremarkable.

$>$ Early thrombolysis in patients with no contra-indications offers the best prognosis.

\section{References}

1 Lazzaro NA, Wright B, Castillo M et al. Artery of percheron infarction: imaging patterns and clinical spectrum. Am J Neuroradiol 2010;31:1283-9.

2 Arauz A, Patino-Rodriguez HM, Vargas-Gonzalez JC et al. Clinical spectrum of artery of Percheron infarct: clinical-radiological correlations. J Stroke Cerebrovasc Dis 2014;23:1083-8.
3 National Institute for Health and Care Excellence. Stroke and transient ischaemic attack in over 16s: diagnosis and initial management: NICE guideline [NG128]. NICE, 2019. www.nice.org.uk/ guidance/ng128 [Accessed 03 April 2021].

4 Obuchowska I, Mariak Z. Internuclear ophthalmoplegia-causes, symptoms and management. Klin Oczna 2009;111:165-7.

5 Amin OSM, Shwani SS, Zangana HM et al. Bilateral infarction of paramedian thalami: a report of two cases of artery of Percheron occlusion and review of the literature. Case Reports 2011;2011:bcr0920103304

Address for correspondence: Dr Charles Badu-Boateng, Frimley Park Hospital, Portsmouth Road, Camberley, Surrey GU12 7UJ, UK.

Email: charles.badu-boateng@nhs.net

Twitter: @C_Badu_Boateng 